\title{
Profile Characteristics of Individuals Eligible for the Air Traffic Controller Training
}

\author{
Ing. David Langr \\ Department of Air Force and Aircraft Technology, Faculty of Military Technology \\ University of defence \\ Brno, Czech Republic \\ davidlangr@europe.com
}

\begin{abstract}
The article deals with the realization of the hypothesis that individuals eligible for the Air Traffic Controller training have a common specific profile, which is a function of the characteristics of individuals engaged in this profession. Defining such a basic profile is based on the results of the experiment realized within the doctoral thesis of the author.
\end{abstract}

Keywords-Air Traffic Controll;selection procedure; aptitudes; simulations; tests

\section{INTRODUCTION}

Air traffic controller profession is a highly professional and mentally demanding job. It is characteristic by work in a complex information environment with high requirements for resistance to fatigue, stress, monotony, but also for other aspects of personal and power dimension of man, as well as aboveaverage personal responsibility. With regard to the responsibility for the safety and continuity of air traffic, individuals chosen for the job should be selected according to the criteria that will be able to reveal the extent of their talents for this type of profession.

\section{SELECTION PROCEDURE}

The selection procedure is necessary to focus on the search for individual professional and personal qualities. Its specific rounds, including the number, may vary slightly in different states of the European Civil Aviation Conference (ECAC). Differences in requirements and approach are also depending in the case of civil or military air traffic service providers. However, the selection process should meet at least the following aspects:

Determining the minimum and maximum age to begin the Air Traffic Controller (ATCO) training;

- $\quad$ Required level of education;

- Aptitudes testing;

- Medical testing;
- Evaluating the language proficiency - English language.

The aim of the selection procedure is to find candidates that are sufficiently mature educationally, physical and mental condition so that they are able to acquire, retain and demonstrate the required relevant theoretical and practical skills.

Logical reasoning offers the hypothesis that individuals eligible for the ATCO training should have a common profile that is a function of the characteristics of individuals performing this profession. The experiment was carried out as part of the doctoral thesis of the author [1], where one of the goals was to find a basic profile of these individuals on the basis of certain common characteristics.

\section{EXPERIMENT}

The experiment involved 58 subjects. They were selected specifically on the basis of certain common characteristics. Profile characteristics and numerical representations are presented in Figure 1. Characteristic "Mensa" means that the subject is a member of Mensa Czech Republic, i.e. his / her IQ is at least 130. Characteristics University (T) means Technical, University $(\mathrm{H})$ means Humanities. 


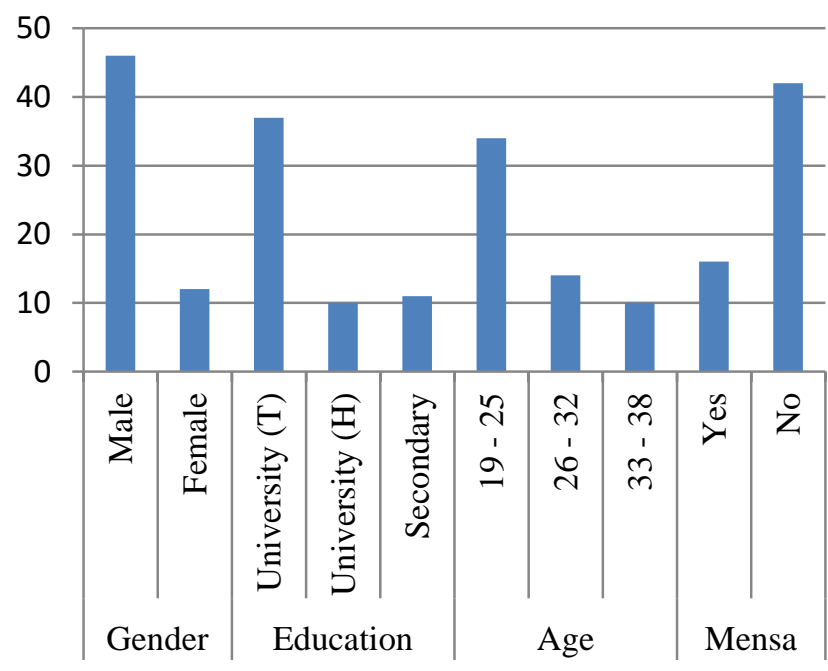

Figure 1. Profile characteristics. [1]

The battery of the professional simulation tasks / tests was focused on the following aptitudes and skills essential for ATCO: memory, spatial imagination, mathematical / numerical reasoning, planning / decision making, multitasking and work under a pressure. Batteries contained a total of 6 sections, each of which focused on one of the following talents:

- "Memory“ test was focused on the ability to take over and for specified time to retain the information associated with the current situation on the radar display, and on the ability to take and store sensory picture of the position of the aircraft. There were a total of four jobs in which subjects screen simulated aerial situation, monitored for 2 minutes, which generally consisted of 12 targets. After the expiration time, subjects were required to enter the flight level of individual goals or sector in which they are located, or both these requirements (depending on specification).

- „Spatial imagination“ test was focused on the subject's ability to orient in three-dimensional space. In this task, they observed static situation including 20 aircrafts for 8 minutes. The aim was to draw the front and side of 10 set aircrafts during these eight minutes.

- „Mathematical / numerical reasoning“ test was testing the individual's ability to perform basic arithmetic operations on the rapid and adequately precise calculation of the horizontal speed, vertical rate of climb / descent and distances. This was a set of tasks divided into two parts. In the first part, 16 static aircraft at different speeds and altitudes were projected on the screen. The task was to solve the given numerous tasks relating to the aircraft or groups of aircraft. In the second part, the aerial situation consisted of 10 static targets displayed for 10 minutes. The task of the subjects in this section was solving two assignments in the order of aircraft in flight to the relevant points.

- „Planning / decision making“ test was focused on the ability to evaluate and select the most suitable alternative solution so that the solution was effective with respect to the further development of the situation and best fulfill the preferences. There were three exercises in which they were shown three different maps composed of two runways and marked points connected with tracks and each other. The assignment was to write the path of aircraft over the points to the desired runway in the order and subject to certain rules. Total time for fulfilling the task was 10 minutes for the first two jobs, and 15 minutes for the third.

- „Multitasking“ test was focused on the subject's ability to perform independent tasks relating to the operation of aircraft at one part of the radar display and simultaneously to monitor and process information from another part of the same radar display. It was a task in which subjects should led 4 aircrafts by commanding headings through 2 corridors without disrupting borders. During this assignment on the screen in chronological order appeared more separate tasks that subjects should have adequately solved. Each of these tasks has been seen only after a certain time. The total task time was 33 minutes.

- „Performance capacity“ test is based on a combination of tests "Memory", "Planning / decision making" and "Multitasking" where the nature of the role played an important role in the subject's ability to make decisions and set priorities under pressure. It was a simulation in which the task of the subject was to manage air traffic in a simplified model of an imaginary airspace. There were a total of 8 aircraft that entered gradually into airspace and that proband directed by the commanding headings. The space was also gradually entered by aircraft, flying along a defined route, which could not be controlled by the subject. The task was to vector handled aircraft in the specified order through several points (also in a defined order). In addition subject had to comply with the rules relating to the loss of separation of aircrafts, disruption of borders of the airspace, entering the restricted area, etc.

\section{RESUlTS}

Evaluation of tests "Memory, spatial imagination, mathematical thinking, planning / decision-making and multitasking" was determined as the ratio of correct answers to the maximum possible, and is expressed as a percentage of success. Performance capacity test evaluation was based on the weighted average achieved positive and negative points.

To calculate the average success rate of subjects / profile group was used the median, which is in contrast to the arithmetic average less sensitive to outliers

The influence of the individual profile characteristics for success in tests is expressed as the average percentage of the group profile. This method, however, is burdened with a certain degree of uncertainty because in case of small number of subjects there can't be determined whether the success of the evaluated profile characteristic is function dependent on the characteristics, or function dependent on a combination of different characteristics which are common in the group, 
because every subject in the group simultaneously belongs into other profiled groups. The influence of this type of solution was in so statistically small number of test subjects, at least partially mitigated by the fact that the results are defined only on those who scored above the overall average. With these subjects there is monitored the frequency of occurrence of individual profile characteristics. Excluding the impact of distortion, caused by the different number of subjects in profile groups, was achieved by means, that the success of the profile group was expressed as a ratio of the group entities who have achieved at least overall average success rate in consideration of the total number of entities of the group.

Figure 2 shows results of each test according to the profile characteristics.

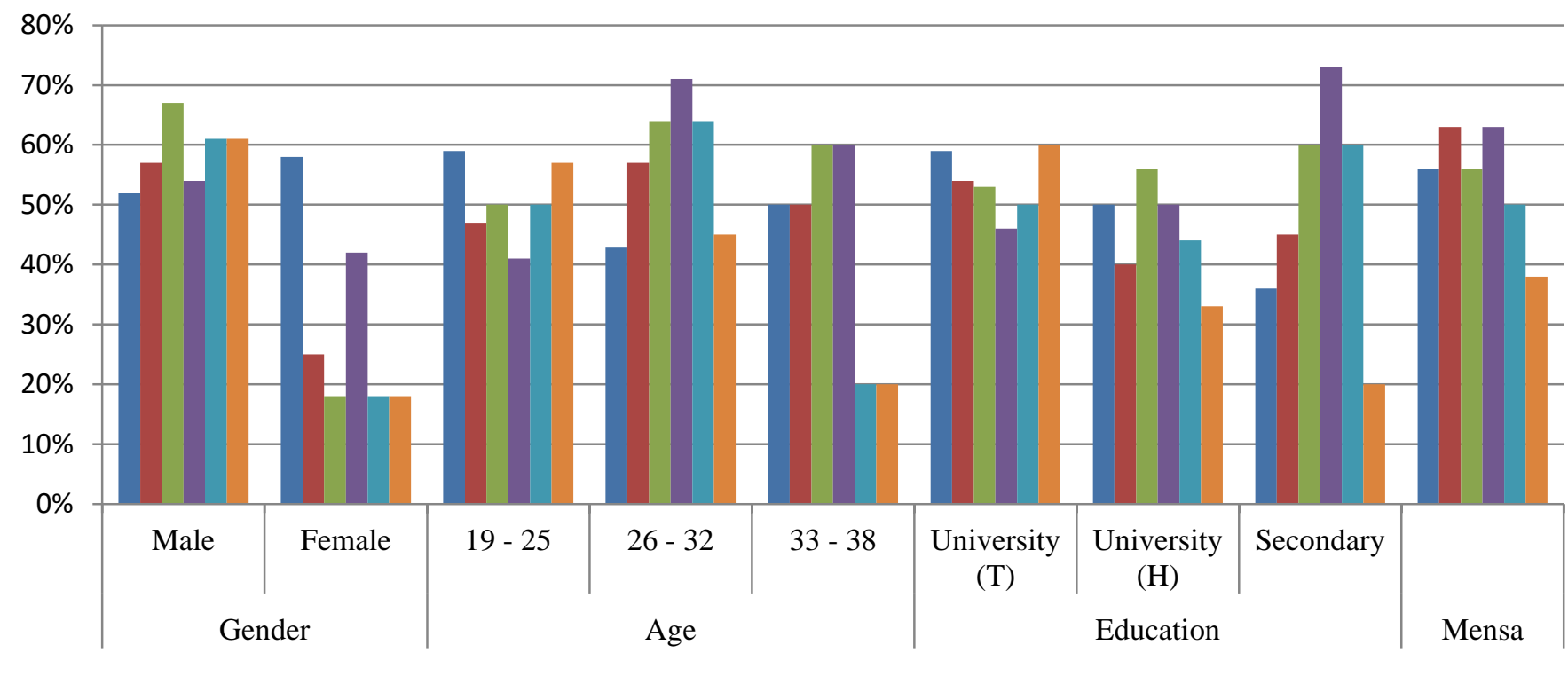

Memory $\quad$ Spatial imagination $\square$ Mathematical

Figure 2. Results of profile groups

Based on the analysis of Figure 2, we are able to determine the characteristics of the profile that evokes a higher probability of success in the selection procedure for the profession of ATCO. Success values are shown in Table 1.

Based on results of the experiment, shown in table 1, we are able to say that the best combination of specific profile characteristic is male, age 26 to 32 with technical university education and above average intelligence.
TABLE I. PROFILE GROUP SUCCESS RATES

\begin{tabular}{|c|c|c|}
\hline \multirow{3}{*}{ Gender } & Male & $59 \%$ \\
\cline { 2 - 3 } & Female & $30 \%$ \\
\hline \multirow{3}{*}{ Age } & $19-25$ & $51 \%$ \\
\cline { 2 - 3 } & $26-32$ & $57 \%$ \\
\cline { 2 - 3 } & $33-38$ & $43 \%$ \\
\hline \multirow{3}{*}{ Education } & University (T) & $54 \%$ \\
\cline { 2 - 3 } & University (H) & $46 \%$ \\
\cline { 2 - 3 } & Secondary & $49 \%$ \\
\hline
\end{tabular}

\section{CONCLUSION}

This article is primarily concerned with some profile characteristics that evoke greater success in the selection procedure for the ATCO job. However, it should be emphasized that the successful completion of the training and thus gain ATCO license is largely influenced by the actual system of theoretical and practical training, i.e. its quality and the 
professional and personal qualities of involved persons. Therefore, solely the selection procedure based on the aptitudes and the profile of the candidates (even if it would be a totally perfect system) can't guarantee the successful completion of the subsequent practical training. This can be achieved only by combining a sufficiently high quality selection system (aptitudes for the profession, psychological profile, language skills, physical fitness) and high quality of theoretical and practical training of ATCO students.

\section{REFERENCES}

[1] Langr, David. Means and methods for supporting the selection and preparation of military specialists of Air Navigation Services (in Czech) [PhD thesis]. Brno: University of defence, 2015. 